\title{
Exploring Weaire-Phelan through Cellular Automata: A proposal for a structural variance-producing engine
}

\author{
André L. Araujo \\ University of Campinas, Brazil \\ a.araujo@fec.unicamp.br \\ Gabriela Celani \\ University of Campinas, Brazil \\ celani@fec.unicamp.br
}

\begin{abstract}
Complex forms and structures have always been highly valued in architecture, even much before the development of computers. Many architects and engineers have strived to develop structures that look very complex but at the same time are relatively simple to understand, calculate and build. A good example of this approach is the Beijing National Aquatics Centre design for the 2008 Olympic Games, also known as the Water Cube. This paper presents a proposal for a structural varianceproducing engine using cellular automata (CA) techniques to produce complex structures based on Weaire-Phelan geometry. In other words, this research evaluates how generative and parametric design can be integrated with structural performance in order to enhance design flexibility and control in different stages of the design process. The method we propose was built in three groups of procedures: 1) we developed a method to generate several fits for the two Weaire-Phelan polyhedrons using CA computation techniques; 2) through the finite elements method, we codify the structural analysis outcomes to use them as inputs for the CA algorithm; 3) evaluation: we propose a framework to compare how the final outcomes deviate for the good solutions in terms of structural performance and rationalization of components. We are interested in knowing how the combination of the procedures could contribute to produce complex structures that are at the same time certain rational. The system developed allows the structural analysis of structured automatically generated by a generative system. However, some efficient solutions from the structural performance point of view do not necessarily represent a rational solution from the feasibility aspects.
\end{abstract}

Keywords: Structural design; Complex structures; Bottom-up design approach.

\section{Introduction}

Complex forms and structures have always been highly valued in architecture, even much before the development of computers. However, as Mitchell, Moore and Turnebull (1993, p. 195) have pointed out, when referring to the Alhambra's complex decorative patterns, "intricacy is more satisfying if built in order". This is true, not only from a perceptual point of view but also in regards to structural analysis and manufacturing issues. For these reasons, many architects and engineers have strived to develop structures that look very complex but at the same time are relatively simple to understand, calculate and build.

Computers have improved structural design substantially since the 1960's. One of the first examples can be noticed in Expo '67 in Montreal when German architects demonstrate industrial and engineering expertise and innovative technology, such as the computers structural analysis (Otto \& Rasch, 1996). After architectural movements including Archigram and Metabolism, structural design experimented significantly with changes in the 1990's, when architectural design practices incorporated software for automobile and aerospace applications. The Guggenheim Museum in Bilbao inaugurated in 1997, is one of the best examples of that time. The complex shape designed by Frank Gehry demanded an intricate structure dimensioned after several physical and virtual models.
From the division proposed by Rowe (1971, p. 68), the structural design approach applied in Guggenheim Bilbao has many top-down aspects, because essentially an overview of the structural system is formulated and then broken down into subsystems. In the last twenty years, the CAD software improvements allow us to optimize these types of structures using an iterative algorithm based on set performance criteria, material properties and fabrication constraints (Fering \& Søndergaard, 2012). Probably in the next few years, the design of complex structures will become increasingly dynamic with the creation of a real-time form finding tools for architects based on engineer's methods (Bialkowski \& Kepczynska-Walczak, 2015). This design approach allows the designer to begin with an inefficient form and then search for a more efficient form by minimizing local bending stresses. According to Kilian \& Orschendorff (2005), there are many analysis tools for refining forms, but there are few design tools for exploring and creating new structural forms.

On another hand, Rowe (1971, p. 71) defines the bottom-up design approach as a piecing together of systems to give rise to more complex systems, thus making the original systems sub-systems of the emergent system. A good example of this approach is the Beijing National Aquatics Centre design for the 2008 Olympic Games, also known as the Water Cube. At the end of the nineteenth century, the mathematician Lord Kelvin proposed a problem in which he inquired how space 
could be partitioned into cells of equal volume with a minimum surface area between them. The Weaire-Phelan structure, developed in 1933 as a result of computer simulations of foam bubbles, is a possible solution for this problem. It is based on a spatial arrangement of two different types of cells, a dodecahedron and a tetrakaidecahedron (Weaire \& Phelan, 1994). The Weaire-Phelan structure was used as inspiration for the Water Cube, in order to generate an irregular composition that resembles bubbles (Pohl, 2008). In this case, the geometry was rotated in two directions before being sliced into its box shape, giving the impression of randomness. While the truss structure shows no variation, the box surface shows some variance in cell sizes and therefore in density (Fischer, 2005), which results in a very complex but at the same time ordered/organized pattern in the façade design (Fig. 1a,b). Obviously, the whole shape of the Water Cube box has much less complexity than Gehry's shapes. However, at a smaller component level, the Water Cube structure could present great levels of the complexity also (Fig. 1a,b).

\section{- Paper450-01.JPG}

Figure 1: Complex structural systems viewed in different scales: (a) Disney Concert Hall, Los Angeles (source: http://www.paperny.com/); (b) Water Cube, Beijing.

Important developments from academic research have provided tools to combine some bottom-up strategies with the refining solution. One of them is Evolutionary Structural Optimization (ESO and after, BESO), that allows the material to be removed and added simultaneously. In this method, the elements with lowest von Mises stresses are removed and void elements near the highest von Mises stress regions are switched on as solid elements (Huang \& Xie, 2010). Arata Isozaki \& Associates have applied ESO to several of their designs. One of the first attempts was a design entry in a competition for a new station in Florence, Italy (Cui et al., 2003). The design entry by Arata Isozaki features a flat roof, supported at several points by organically shaped columns, designed through the use of ESO (Veenendaal, 2008).

Currently, researchers are using more global shape optimization than explored building blocks interaction, because the last ones are commonly standardized, and this represents a limitation in the form finding process. Assuming that the recent improvements in computational modeling could approach the bottom-up generative systems to the same formal freedom of top-down strategies, some advantages arise, mainly related to the structural manipulation in the component level before getting to the global shape.

This research aims to explore a design method to generate structural elements from the bottom-up point of view. Using the Weaire-Phelan geometry, we proposed a generative system based on Cellular Automata (CA) in order to obtain structural portal frames. After that, we evaluated the feasibility aspects with computational simulations comparing results with the predefined frames. Besides this, we discuss what possibilities could be opened to the structural design from this point of view.
Thus, we organized the procedures into three groups:

i) General design procedure: we developed some algorithmic tasks to generate several fits for the two Weaire-Phelan polyhedra using CA computation.

ii) FEM Analyses: through the finite elements method, we propose a framework to compare how the final outcomes deviate for good solutions in terms of structural performance;

iii) Post-analysis exploration: we propose some intervention in the Weiare-Phelan blocks, in order to verify impacts from component manipulation in the whole structure.

\section{General Design Procedure}

We start with two layers of Weaire-Phelan geometry organized on directions $X$ and $Z$. In the $X Z$ plane, two irregular pentagonal dodecahedra (12-sided) and six tetradecahedra (14-sided) are packed with a periodic lattice. The dodecahedra do not touch each other but are surrounded by tetradecahedra. In order to manipulate each set of polyhedra, we determine their center points and place them on regular grids of 9 by 5 . After that, we create a reference to each type of polyhedron, based on their position on the layer. For instance, the D1 means dodecahedron on the front layer and $\mathrm{T} 2 \mathrm{C}$ refers to a tetradecahedron on the back layer, which is facing up in relation to the reference face. Besides this, we separated each set using the RGB colors palette. We define that each polyhedron could be moved and occupy a place in its own layer (Fig. 2).

\section{- Paper450-02.JPG}

Figure 2: Weaire-Phelan organization.

\section{Variance-producing engine}

The A cellular automaton is a collection of "colored" cells on a grid of specified shape that evolve through a number of discrete time steps, according to a set of rules based on the states of neighboring cells. The rules are then applied iteratively for as many time steps as desired (Wolfram 2002, p. 48). A 2-dimensional CA consists of a regular square of cells. Each cell is updated in discrete time steps according to a rule that depends on the value of sites in the neighborhood around it. The game of life is a type of 2D-CA invented by John H. Conway and popularized in the 1970's, in which the cells can be either alive or dead (Gardner, 1970). The status of each cell depends on the status of that cell's 8 neighbors. The initial pattern is the first generation. The second generation evolves from applying the rules to every cell on the game board. Afterward, the rules are iteratively applied to create future generations. For each generation of the game, a cell's status in the next generation is determined by a set of rules. Using the grid of polyhedron center points we define these rules:

i) any live cell with fewer than two live neighbors dies;

ii) any live cell with two or three live neighbors lives, unchanged, to the next generation;

iii) any dead cell with exactly three live neighbor cells will come to life. 
SIGraDi 2016, XX Congress of the Iberoamerican Society of Digital Graphics 9-11, November, 2016 - Buenos Aires, Argentina

iv) any live cell with more than three live neighbors dies.

From these rules, we can produce a large number of arrangements, and control the position of each polyhedron within them.

\section{Portal frames results}

In the investigation of CA as a portal frame generator, we limited the number of the life-forms, in order to restrict the search field. After a primary exploration, we defined five initial generations: $A, B, C, D$ and $E$, in order to record their results. The points arranged in a regular lattice represent a portal frame and it was proposed two restraints to create a portal frame shape: 1) span: at the bottom horizontal axis, at least five cells should be dead; and at the central vertical axis at least four cells should be dead; 2) continuity: solutions that presented gaps between polyhedrons were rejected. After establishing those conditions, four possible solutions filled these requirements and were then considered (Fig. 3).

\section{- $\quad$ Paper450-03.JPG}

Figure 3: Life-forms iteration and portal frames obtained.

After these life-form combinations, there were four Portal Frames generated (PF) using different quantities of polyhedrons: PF1= 73; PF2 = 57; PF3 = 49; PF4 = 44. We observed that some life-form combinations moved toward or away from stiff forms. Although we just consider four cases to analyze, these lives produced a great number of solutions, because their initial configuration continues the iterations for a long time before death. In the same way, the search field limitation means a great reduction of the number of possible combinations, and this allows us to have an estimate of how many solutions could be generated through this generative system.

Whether polyhedrons are individually evaluated or not, the dodecahedron would present a better stability level than tetradecahedron because it has double symmetry and equal faces. Naturally, it is expected that the PF1 presents a lower displacement in the $\mathbf{Z}$ direction because it has more structural elements, smaller free span, and 16 dodecahedrons. The PF2 and PF3 look like a traditional portal frame of straight columns and beams. The PF4 has a curved structure similar to an arc and commonly this represents an advantage to support loads above it.

The most common form of portal frame used in the construction industry is the pinned base frame with different rafter and column member size. In order to compare the results, we define the basic structural form of the portal frame using the Weaire-Phelan definition as a comparative case. Basically, the analysis method consists of defining the load that can be applied to the frames so that we can observe effects of axial force, shear, buckling, and so on.

Through Finite Element Method (FEM) in Karamba3D® we define a basic set of procedures that cover: (a) create a portal frame structure composed of bars and nodes from the edges and vertices of each polyhedron, removing duplicated lines and points; (b) define a standard cross section of circular hollows: $\varnothing 73.0 \mathrm{~mm}$; thickness: $3.6 \mathrm{~mm}$; (c) define material: steel ASTM A 501, fy $=250 \mathrm{MPa}$ and fu $=400 \mathrm{MPa}$; (d) establish a range of loads: gravity, point and uniform load; wind pressure (e) create supports at nodes, based on their coordinates.

In order to perform comparisons between analysis results, a consideration of connection stiffness is necessary. However, the nature of the connection stiffness is unknown. Further, it was not intended in this research to experimentally determine the connection stiffness associated with the frames studied herein. Although several outcomes had been obtained, the same load conditions were applied to evaluate the displacements.

In a typical design process, the frame structure is designed based on two factors: strength and stiffness. Member stiffness affects the stability and displacement of the structure. Among generated frames, the PF2 presented the worst situation, having a high displacement in the middle of the span and also high levels of horizontal displacement. Considering the structural mass, the PF1 does not present a good solution as well, because it used a large number of elements and more than twice the displacement when compared to the comparative case. The best solutions are PF3 and PF4. On one hand, PF3 has the advantage of spending fewer elements than PF4; in another, PF4 has the advantage to provide a larger free span.

In order to explore the solution, we choose the PF1 to investigate ways to improve the structural aspects through the manipulation of the life-forms. With this goal in mind, we identify regions on the structure with high displacement and investigate ways to minimize them.

\section{Results and Discussion}

Architectural design processes have been increasingly tailored to provide more precise considerations of context in the generation and optimization. In the structural design context, it is not simple to establish a negotiation between structural performance, materials, environment variables and functional intentions. The early stages of design have incorporated more and more knowledge. In this sense, the design expectations do not concern only integrating subjects. Besides, there is a necessity of frameworks in which the generative systems can produce several efficient solutions, not only the optimal one. In the relationship between topdown and bottom-up design approaches the thinking about component levels could bring an important contribution to the complex shapes designed. At last, more investigations about CA features, with its potentials, could link it to other computational design tools, making it more useful and faster in decision processes.

Using the same rules and life-forms, we define simple conditions to insert additional polyhedrons in those regions. Again, the 8 colored layers were used to represent the frame in which each polyhedron could be placed. Using loops in the $\mathrm{C \#}$ program language we define instances where codes need to be repeated until a certain condition is met, in this case, when high displacements are identified. To address this need, C\# provides the while loop. Each time the while loop is executed, it checks the condition in which the variable dispPt 
is lower than $0.95 \mathrm{~m}$, and after that, it decides whether to add the new polyhedron or not. When the while counter reaches 44 ( 9 by 5 points at the grid, included slot position 0 ), the condition becomes false and the loop ends. Otherwise, when the displacement approaches zero, it's possible to delete some elements. In a situation of a uniformly distributed load, it is possible to express that condition from the number of polyhedra per unit volume (Fig. 4).

\section{- Paper450-04.JPG}

Figure 4: Structural improvements using conditionals.

In the design process with more top-down characteristics, part of the role of the digital technology has been to search for simplicity within geometric complexity, and to bring the description of the building elements with the conventional language of contemporary construction practice. On another hand, we proposed a bottom-up structural design system, which is necessary to provide a complete, or at least extensive, description of the structural components: bars, angles, connections, and so on. Although other alternatives of elements could be defined beforehand, the relations among these components are systematically identified and established. Therefore, the role of the CA generative systems, in this case, is to combine the predefined components, and not allow changes in the standard definitions throughout the iteration.

Under these circumstances, we provide some alternatives to the generative systems within a co-rationalization context. This approach offers various opportunities for design in the compositional details and the external shape of an apparently irregular structure, while rationalizing it simultaneously. Individual polyhedra can be added, deleted or moved without disturbing the overall logic of the configuration. In another way, post-rationalization context commonly consists in the search for improving the structural performance even when the structural shape is inefficient. In a real case, the negotiation between structural performance and other disciplines in the design process has been established, and consequently, the greater emphasis of structural aspects in the design context could define the choice for a bottom-up form-finding system.

After define continuity one of the requirements of the CA iteration, we took one of those four portal frames to exemplify the possibility of manipulation in the co-rationalization context. In general, the combination of the edges of 14-side and 12-side polyhedra guarantee a stable geometric configuration, since continuity exists between bars and nodes. Among the portal frames found from the CA generative system, the more efficient in terms of structural performance in a given context of loads could be improved from automatic or manual procedures. Initially, the Portal Frame 1 (PF1) generated exclusively by the CA interaction presents some structural instability due to the axial tension checked under the load proposed. Despite the FEM analysis and of the automatic loops, this scenario could be improved by inserting, moving or deleting polyhedra in the portal frame by the heuristics. In other words, it's possible to stop the iteration, insert, delete or move any polyhedron, and, after that, keep running the CA iteration.

After some structural fixes, we generated 4 other portal frames based on the geometry of the PF1. The locals of the manual or automated intervention were locked and we kept the CA intervention in other parts of the structure. It opens the opportunity to construct a tridimensional structure from two assumptions: searching for the organization for its parts and searching for improving the solution from the interventions. This is not just a full optimization commonly made after the whole shape definition. In this case, the designer knows previously that the standard components promote stability for the global solution and the small fixes could be made in the structure chimes with other design issues.

\section{- Paper450-05.JPG}

Figure 5: Variance-producing engine iteration to generate four portal frames; after structural improvements, more iteration to generate more portal frames.

When we define some requirements in the generative system, we are highlighting some characteristics for the structural shape and, consequently, for the whole architectural form as well. However, when we keep the structural arrangement we eliminated any improvisation in the structural solution. As much as the design techniques were improved in order to manipulate standard blocks or components, the bottom-up design process could have more formal freedom. In the computational design, the cellular automata are used many times without clear proposals or goals, producing unexpected solutions, but away from the good solutions. As a variance-producing engine, the cellular automata works well because the generative system does not have to compromise to generate the solution straight forward. In other words, the top-down decision represented here for the requirements, contributes to driving the solution in a global context while the link between standard blocks and the generative systems guarantee variance and quality in the local context.

In this study, the bottom-up design model provides the possibility to manipulate structures on the component levels. From this point of view, many useful alternatives could be obtained before a more careful structural analysis. Despite the model being presented and tested in a rectangular grid, the polyhedra can be handled in other plane surfaces. Furthermore, the model has a good potential to be used in other applications, including 3-dimension portal frames and more complex structures, mainly when the structure has an important role in the architectural environment. This will be considered in future works.

\section{Conclusion}

Architectural design processes have been increasingly tailored to provide more precise considerations of context in the generation and optimization. In the structural design context, it is not simple to establish a negotiation between structural performance, materials, environment variables and 
SIGraDi 2016, XX Congress of the Iberoamerican Society of Digital Graphics

9-11, November, 2016 - Buenos Aires, Argentina

functional intentions. The early stages of design have incorporated more and more knowledge. In this sense, the design expectations do not concern only integrating subjects. Besides, there is a necessity of frameworks in which the generative systems can produce several efficient solutions, not only the optimal one. In the relationship between topdown and bottom-up design approaches, the thinking about component levels could bring an important contribution to the complex shapes designed. At last, more investigations about CA features, with its potentials, could link it to other computational design tools making it more useful and faster in decision processes.

\section{Acknowledgments}

The authors would like to thank the grants 2012/05475-4 and 2015/01228-0, São Paulo Research Foundation (FAPESP).

\section{References}

Bialkowski, S. \& Kepczynska-Walczak, A. (2015). Engineering Tools Applied in Architecture: Challenges of topology optimization implementation. ECAADE, Vienna, 261-268.

Cui, C. et al. (2003). Computational morphogenesis of 3D structures by extended ESO method. Journal of the International Association for Shell and Spatial Structures, 44(141), 51-61.

Feringa, J. \& Søndergaard, A. (2012). Design and Fabrication of Topologically Optimized Structures: an integral approach: a close coupling form generation and fabrication. ECAADE, Prague, 495500
Fischer, T. (2005). Generation of Apparently Irregular Truss Structures, CAADFutures, Vienna, 229- 238.

Gardner, M. (1970). The fantastic combinations of John Conway's new solitaire game "life". Scientific American, 4(223), 120-123.

Huang, X., \& Xie, Y. (2010). Comparing BESO with Other Topology Optimization Methods. In Huang, X., \& Xie, Y., Evolutionary Topology Optimization of Continuum Structures: Methods and Applications (pp. 51-62). London: Wiley.

Kilian, A. \& Ochsendorf, J. (2005). Particle-spring for structural formfinding. Journal of the International Association for Shell and Spatial Structures, 46(148), 77-84.

Moore, C., Mitchell, W. and Turnbull, W. (1993). The Poetics of Gardens, The MIT Press, p. 195.

Otto, F. \& Rasch, B.: 1996, Finding Form: Towards an architecture of the minimal, Axel Menges.

Pohl, B.: 2008, Watercube: the book. Actar-D.

Rowe, P.: 1971, Design Thinking, The MIT Press.

Veenendaal, D.: 2008. Evolutionary Optimization of Fabric Formed Structural Elements. Master thesis, Delft University of Technology.

Weaire, D. \& Phelan, R. (1994). A counter-example to Kelvin's conjecture on minimal surfaces. Philosophical Magazine Letters, 2(69), 107-110.

Wolfram, S.: 2002, A New Kind of Science. Champaign, Wolfram Media. 